\title{
Cathepsin-B and Caveolin-1 Gene Expressions inOral Lichen Planus and Oral Squamous Cell Carcinoma
}

atessa pakfetrat

Mashhad University of Medical Sciences

zahra delavarian

Mashhad University of Medical Sciences

nooshin mohtasham

Mashhad University of Medical Sciences Ghaem Hospital

farnaz mohajertehran

Mashhad University of Medical Sciences

Negin Samiee ( $\nabla$ negin.samiei@gmail.com )

Kurdistan University of Medical Sciences https://orcid.org/0000-0002-3929-0098

\section{Research Article}

Keywords: Oral lichen planus, OSCC, cathepsin-B, caveolin-1, real-time PCR

Posted Date: May 12th, 2021

DOI: https://doi.org/10.21203/rs.3.rs-461746/v1

License: (9) This work is licensed under a Creative Commons Attribution 4.0 International License. Read Full License 


\section{Abstract}

\section{Background}

Given the importance of the role of gene expression in the prognosis and diagnosis of malignancies of the head and neck, we examined in this study the gene expressions of cathepsin-B (Cat-B) and caveolin-1 (Cav-1) in oral squamous cell carcinoma (OSCC) and oral lichen planus (OLP) and compared them to controls. We also investigated their relationship to clinicopathological indices.

\section{Methods}

In this case-control study, 29 biopsy samples from OLP patients were compared to 29 paraffin blocks of OSCC samples as well as 28 paraffin blocks of normal oral tissue. The mean age of OSCC and OLP patients were $59.24 \pm 15.04$ and $48.79 \pm 14.17$ years, respectively. Clinical data were analyzed for their relationship to gene expressions using parametric and nonparametric tests.

\section{Results}

The gene expressions of Cat-B and Cav-1 were significantly higher in OSCC and OLP patients compared to controls $(p<0.001)$. The highest expression were found in OSCC, which was significantly different from OLP and controls. There were also significant differences between OLP and control in terms of the gene expression of Cat-B and Cav-1 .There was no association between the gene expression and age, gender, duration of disease, Thongprasom score, smoking and cutaneous lichen planus. However, the expressions were related to the grade and stage of OSCC lesions (p:0.01, p:0.02).

\section{Conclusion}

The gene expressions of Cat-B and Cav-1 in OSCC were associated with the stage and grade of lesions. Therefore, they appear to be useful in predicting the biological behavior of OSCC and malignant transformation of OLP.

\section{Introduction}

Oral Lichen Planus (OLP) is a chronic mucocutaneous disease that affects 0.5 to $2.2 \%$ of adults. It is considered, however, as a premalignant condition with the potential to transform into oral squamous cell carcinoma (OSCC), i.e. a malignant condition. OSCC is the most common malignancy of the oral cavity with more than $90 \%$ of incidence, affecting $1.1 \%$ of adults with or without predisposing risk factors (1). Although the etiology of OLP is unknown, it has been shown that the immunological response due to band-like T-lymphocyte infiltration and the subsequent activation of these cells against antigens of the basal epithelial layer is responsible for (1). The role of cytokines, enzymes and oxidative imbalance in the pathogenesis of OLP has been established or is currently being investigated. 
In OLP, the accumulation of oxidative stress in the basal layer causes hypoxia in the lower stroma, which lowers the $\mathrm{pH}$ of the tissue and consequently increases some enzymes in the extracellular matrix and epithelial cells. This leads ultimately to the spread of the lesion as cell connections and extracellular matrix components are lost (2). Extracellular enzymes, cell surface molecules, and immune response are also involved in the development and spread of oral cancer. According to the epithelium-connective tissue model, the differentiation and maturation of mucosal epithelial cells is the result of the communication between epithelial and connective tissue cells due to enzymes, molecules and growth factors that play a similar role in the carcinogenesis (1).

The effect of enzymes and surface molecules on the disease process and its biological behavior, prognosis and metastasis, the possibility of malignant transformation, especially in precancerous lesions, have been investigated in various studies. Both Cat-B as an extracellular enzyme and Cav-1, as a structural protein molecule in cell membranes, are involved in carcinogenesis.

As a lysosomal cysteine protease, cathepsin-B (Cat-B) is initially an inactive proenzyme, which becomes active and matures at low pHs. This enzyme degenerates the extracellular matrix by converting prourokinase-type plasminogen to urokinase-type. It is an important biomarker for OSCC (5) as it activates other proteases, particularly matrix metalloproteinases (MMPs), in OSCC as well as in other cancers (8).

Caveolins, on the other hand, are a family of proteins whose potential role in reducing oxidative stress in tissues has recently been examined. These protein microstructures aggregate into caveolae structures in the cell membrane. Due to the inverse relationship between the amount of this protein and oxidative stress in tissue, we expect a decrease in Cav-1 expression in various cancers. Increased expression of p53 tumor suppressor gene has been linked to decreased expression of Cav-1 (7).

Many studies view the gene expression of Cav-1 as an oncogene, as well as an indicator for malignancy, angiogenesis and differentiation, clinical stage of the tumor, metastasis and response to chemotherapy. In addition, an increased expression of Cav-1 has been observed in malignant salivary conditions, melanoma, esophageal SCC and, on the contrary, as tumor suppressor genes in ameloblastoma, ovarian carcinoma and various sarcomas. Different functions for different parts of the Cav-1 protein have therefore been proposed to address this dichotomy.

Given the nature of OLP as a precancerous disease with the potential for transforming into malignancy, as well as the role of enzymes as predictive markers of cancer biological behavior in early diagnosis, determining various behaviors and cellular changes, the severity of the disease, the possibility of metastasis and prognosis, we investigated in this study the gene expressions of Cat-B and Cav-1 in OLP and OSCC.

\section{Materials And Methods}


In this case-control study, tissue samples from 90 patients were initially examined in three groups of 30 each. Four samples were excluded either due to their poor quality for PCR or due to the location of the OSCC outside the oral cavity. In the end, 29 tissue samples with OLP, 29 with OSCC and 28 with fibroid lesions without superficial epithelial hyperplasia and inflammatory infiltration in the connective tissue were included in the case and control groups, respectively. Inclusion criteria were confirmed OLP according to the modified WHO standard (10), OSCC and histopathologically-confirmed fibromas. Exclusion criteria were distorted samples, lichenoid reaction samples, fibroma samples with superficial epithelial hyperplasia and inflammatory inflammation in connective tissue.

\section{Sample Selection}

After obtaining informed consent and during the biopsy, a checklist was recorded with the patient's age, medical history, exact locations of intraoral involvement, and lesion severity according to the Thongprasom scoring (11). In the case of OSCC, the available samples in the archives of the pathology laboratory of the Department of Dentistry were used. After the OSCC samples were confirmed, the blocks were selected for RNA extraction based on the quality and quantity of the tissue, and the grade of lesions was determined by a pathologist according to the invasion of the connective tissue by cancer cells. The stage of the lesions was then determined according to the TNM table (1), the size of the lesion, the involvement of regional lymph nodes and the distant metastasis. The sequence of the glyceraldehyde-3phosphate dehydrogenase (GAPDH) reference gene and the Cav-1 and Cat-B target genes are shown in Table 1.

Table 1

Primer sequence of Cav-1, Cat-B, and GAPD

\begin{tabular}{|c|c|c|c|c|c|c|c|}
\hline Group & $\begin{array}{l}\text { Age } \\
\text { (mean } \pm \\
\text { SD) }\end{array}$ & $\begin{array}{l}\text { Gender } \\
(\mathrm{m}: \mathrm{f})\end{array}$ & $\begin{array}{l}\text { Grade } \\
(\%)\end{array}$ & $\begin{array}{l}\text { Stage } \\
(\%)\end{array}$ & $\begin{array}{l}\text { Thongprasom } \\
\text { score }\end{array}$ & $\begin{array}{l}\text { Cav-1 } \\
\text { expression } \\
(\%)\end{array}$ & $\begin{array}{l}\text { Cat-B } \\
\text { expression } \\
(\%)\end{array}$ \\
\hline $\begin{array}{l}\text { OLP } \\
(29)\end{array}$ & $\begin{array}{l}48.79 \pm \\
14.17\end{array}$ & $9: 20$ & $\begin{array}{l}1: 12 \\
(41.4)\end{array}$ & $\begin{array}{l}1: 12 \\
(41.4)\end{array}$ & $3.17 \pm 1.31$ & $26(89.7)$ & \\
\hline $\begin{array}{l}\text { Control } \\
\text { (28) }\end{array}$ & $\begin{array}{l}49.25 \pm \\
16.44\end{array}$ & 9:19 & $\begin{array}{l}2: 14 \\
(48.3)\end{array}$ & $\begin{array}{l}2: 17 \\
(58.6)\end{array}$ & - & $2(7.1)$ & 1 (3.6) \\
\hline $\begin{array}{l}\text { OSCC } \\
\text { (29) }\end{array}$ & $\begin{array}{l}59.24 \pm \\
15.04\end{array}$ & & $\begin{array}{l}3: 3 \\
(10.3)\end{array}$ & - & - & 27 (93.1) & 27 (93.1) \\
\hline Total & $\begin{array}{l}54.17 \pm \\
15.84\end{array}$ & & - & - & - & $56(48.7)$ & 55 (48.7) \\
\hline
\end{tabular}

To evaluate the gene expression of Cav- 1 and Cat-B, 10 paraffin blocks of 5 to $10 \mu \mathrm{m}$ were first prepared from each sample. They were then deparaffinized with pure xylene and ethanol. A high purity RNA paraffin kit (Roche $\mathrm{GmbH}$, Germany) was used to extract RNA according to the manufacturer's instructions. Next, the cDNA was prepared from the previously extracted RNA using the RevertAid First Strand cDNA Synthesis Kit (Thermo Fisher Scientific Inc., USA). 
Real-time quantitative PCR was performed to determine the expression of Cat-B and Cav-1 using the SYBR Green PCR Master Mix (Thermo Fisher Scientific Inc., USA) on an ABI thermal cycler (One Step Inc., USA) with suitable primers in Table [1]. Differential expression was analyzed using the delta-delta comparison threshold $(\Delta \Delta \mathrm{Ct})$ method. The housekeeping gene GAPDH was used as a reference.

\section{Statistical analysis}

SPSS version 18 was used for statistical analysis and a significance level of 0.05 was considered. The Kolmogorov-Smirnov test was used to examine the distribution of the data, and analysis of variance and the chi-square test were then used to examine the homogeneity of age and gender. For the first group, chisquare and Kruskal-Wallis tests were used to assess qualitative and quantitative gene expressions, respectively, while the Mann-Whitney test was used to assess the quantitative gene expression in the second group. Linear regression was used to adjust the effect of age and gender.

\section{Results}

In this study, the mean age in the OSCC group was $59.24 \pm 15.04$ years and it was $48.79 \pm 14.17$ in the OLP. Moreover, $72 \%$ of the OSCC ( $<0.001)$ and $32 \%$ of the OLP patients $(p=0.01)$ were male. The groups differed significantly in terms of age $(p=0.01)$, so that people in the OSCC group were older than those in the other two groups. In addition, $68.0 \%$ of the OLP patients were female, which was more than the other two groups. Therefore, the three groups were gender-inconsistent $(p=0.002)$. The mean duration of OLP was $17.66 \pm 10.69$ months (Table 2).

Table 2

sample data.

\begin{tabular}{|ll|}
\hline Primer & Sequence \\
\hline Cav-1 Forward & 5'-AACCTCCTCACAGTTTTCATCC-3' \\
\hline Cav-1 Reverse & 5'- CTTGTTGTTGGGCTTGTAGATG-3' \\
\hline Cat-B Forward & 5'-CAGATGAGGCCGTGGCTTAT-3' \\
\hline Cat-B Reverse & 5'-GGGAGTCACATCCAACAGCAA-3' \\
\hline GAPDH Forward & 5'-GGGCTGCTTTTAACTCTGGTAA-3' \\
\hline GAPDH Reverse & 5'-ATGGGTGGAATCATATTGGAAC-3' \\
\hline
\end{tabular}

The most common sites of OSCC and OLP lesions were the tongue (55\%) and buccal mucosa (93\%), respectively, although more than half of the lesions were on the tongue. $58.6 \%$ of OSCC patients were in the second stage (58.6\%) and $48.3 \%$ had second grade lesions (48.3\%). In addition, most of the OLP patients had lesions with a Thangprasom score of 3. Cat-B did not express in most of the samples in the controls (96.4\%), while it expressed in $89.7 \%$ and $93.1 \%$ of the OLP and OSCC samples, respectively. The three groups differed significantly in terms of Cat-B expression $(p<0.001)$. Also, Cav-1 did not express in 
most of the samples in the controls (92.9\%), while it expressed in $89.7 \%$ and $93.1 \%$ of the OLP and OSCC samples, respectively. The three groups also differed significantly in terms of Cav-1 expression $(p<0.001)$ (Table 2).

Pairwise comparisons showed that the expressions of Cat-B and Cav-1 were significantly higher in OSCC samples compared to others. Moreover, the OLP and control samples differed significantly in terms of gene expression (Figs. 1 and 2).

The gene expressions of Cat B and Cav-1 were associated in the OLP $(p<0.001)$, control $(p=0.01)$, and OSCC samples $(p<0.001)$. The association was stronger in OSCC than in the others. There was also no association of Cat-B and Cav-1 expressions with age, duration of disease, and Thongprasom score in the groups $(p<0.05)$. The expression of Cav- 1 was only significantly associated with gender in the controls and was higher in women $(p=0.04)$. Smoking and cutaneous lesions in OLP also had no significant relationship to the expressions of Cat-B and Cav-1 $(p<0.05)$. In OSCC, the mean expressions of Cat-B and Cav-1 were significantly associated with the grade of lesion ( $p=0.01$ and $p=0.003$, respectively) as well as with the its stage ( $p=0.02$ and $p=0.01$, respectively) (Table 2$)$. In contrast to Cav- 1 , whose expression increased gradually with the increase in the grade and stage of lesion, the expression of Cat-B was lower in grade 2 than in grade 1.

Table 3. Relationship between the gene expressions of Cat-B and Cav-1 (mean \pm SD) and the stage and grade of lesions in OSCC (*: Kruskal-Wallis test, **: Mann-Whitney test, ***: Independent t-test).

Table 3

Relationship between the gene expressions of Cat-B and Cav-1 (mean \pm SD) and the stage and grade of lesions in OSCC (*: Kruskal-Wallis test, $* \star$ : Mann-Whitney test, $* \star \star$ : Independent t-test).

\begin{tabular}{|c|c|c|c|c|}
\hline Group & & & Cat-B & Cav-1 \\
\hline \multirow[t]{7}{*}{ OSCC } & grade & 1 & $4.77 \pm 3.42$ & $4.92 \pm 4.41$ \\
\hline & & 2 & $3.26 \pm 7.58$ & $8.31 \pm 4.25$ \\
\hline & & 3 & $12.57 \pm 1.17$ & $20.58 \pm 5.05$ \\
\hline & & $P$ value & $P=0.01 *$ & $P=0.003^{*}$ \\
\hline & stage & 1 & $4.99 \pm 3.53$ & $4.94 \pm 3.2$ \\
\hline & & 2 & $8.3 \pm 3.46$ & $10.51 \pm 6.97$ \\
\hline & & Result & $P=0.02^{\star \star *}$ & $\mathrm{P}=0.01 \star \star$ \\
\hline
\end{tabular}

Linear regression was used to determine the effect of age and gender on gene expression. Because of the role of gender, the regression was performed separately for men and women. After adjusting the effect of gender in men, the expression of Cav-1 in OLP was 2.58 units higher compared to controls, although the 
difference was not significant. In OSCC, on the other hand, the mean expression of Cav-1 was 6.34 units higher than that in controls, and the difference was significant $(p<0.001)$. Therefore, age was not associated with Cav-1 expression.

Similarly, in women and after adjusting the effect of age, the expression of Cav-1 in OSCC was 2.11 units higher than in controls, and the difference was significant ( $p: 0.05)$. The mean expression of Cav-1 in OSCC was 12.56 units higher than that in controls, and the difference was significant $(p<0.001)$. Therefore, age was not associated with Cav-1 expression in OSCC in women.

By adjusting the effect of age and gender, the expression of Cat-B in the OLP samples was 2.68 units higher than the controls, and the difference was significant $(p<0.05)$. Age and gender were not related to the expression of Cat-B.

\section{Discussion}

In this study, the gene expressions of Cat-B and Cav-1 were significantly higher in the OLP and OSCC samples compared to the controls. They were also higher for OSCC than for OLP, along with an increase in the stage and grade of the lesions. Unlike Cat-B, such an increase was gradual for Cav-1. The gene expressions of Cat-B and Cav-1 in OLP were not associated with clinical indices of the lesion, including its severity as per Thangprasom scores and duration, as well as smoking and the presence of cutaneous lichen planus. In addition, the expressions were independent of age and gender. There was a correlation between the gene expressions of Cat-B and Cav-1 in OSCC and OLP, and the correlation was more significant in OSCC.

Given that OLP is inherently recognized as an oral malignancy and OSCC treatment varies based on clinical stage, pathology, and metastasis, determining the behavioral evolution of these lesions is fundamental in choosing the best treatment. Numerous studies have been carried out on various markers for their possible role as predictors of malignant OLP changes as well as on the OSCC prognosis in order to predict their behavior.

In a 2010 study, Yang et al. examined the expression of Cat-B in tissue samples from 30 OSCC patients using three methods, namely immunohistochemistry (IHC), RT-PCR and the Western blot analysis. According to their study, the gene expression of Cat-B was associated with metastasis to regional lymph nodes (5).

Also in 2012, Chen et al. compared changes in the gene expression of Cat-B with the clinicopathological indices of 444 patients with OSCC and found a significant relationship between a single nucleotide pleomorphism (SNP) of Cat-B and an increased risk of OSCC. They also reported a significant relationship between the expression of Cat-B and the grade and stage of lesions in OSCC patients, although this increase was not gradual for the grade (12). 
Satelur (2017) examined the expression of Cat-B in 50 OLP patients as a case group along with 10 OSCC patients and 10 healthy individuals as a control group by IHC. The Cat B-associated staining intensity in the stromal region and basal cells of both lesions was similar and confirmed the role of this marker in OSCC carcinogenesis in the epithelial-connective tissue model. On the other hand, the gene expression of Cat-B in connective tissue as a transmitter of degenerating signals to epithelial cells was included in the pathogenesis of OLP and in the possibility of malignancy. However, no study has been conducted on the relationship between the clinical indices of OLP as well as the clinicopathological indices of OSCC and the gene expression of Cat-B (13).

The gene expressoin of Cav-1 in oral tissues has been shown in various studies to affect the development of OSCC from dysplasia, which is consistent with the clinicopathological indices of OSCC. Hung et al. (2003) immunohistochemically examined 96 tissue samples, including normal oral tissue, precancerous lesions, OSCC tissue and its metastatic type. However, they did not confirm the effect of Cav-1 expression in the primary OSCC lesions and their metastatic type. They also reported a significantly lower Cav-1 expression in metastatic types as well as no association of Cat- 1 expression with age, gender, smoking, stage and grade of the lesions (8). In the present study, the clinical indices including age, gender, severity and duration of lesions had no effect on gene expression in OSCC. However, gene expression was significantly associated with the stage and grade of the OSCC lesions.

Kato et al. (2020) immunohistochemically examined the gene expression of Cav- 1 in tissue samples from 80 OSCC patients and compared it to 15 samples from metastatic lymph nodes and showed that there was a significant association between the expression of Cav-1 in OSCC and metastasis of cell differentiation and lesion invasion. They therefore suggested to use Cav-1 expression for the prognosis of OSCC (14).

There is still some controversy about the relationship between the gene expression of Cav-1 and the stage and grade of lesions in OSCC. According to some studies, as the stage and grade of the lesion increase, so does the expression of Cav-1. Even the expression of this gene has been inversely related to the survival of OSCC patients $(7,15)$. In the study by Routray et al., however, the increase in Cav-1 expression was linked to an improved prognosis (6).

Ashkavandi reported the gene expression of Cav-1 in the cytoplasm of 81 biopsy samples from various oral lesions including hyperkeratosis, dysplastic epithelium, OSCC and OLP, with the gene expression of Cav-1 only being significant in OSCC (92\%), while being $4 \%$ in OLP and increased proportionally with hyperkeratosis to dysplastic epithelium and OSCC (7). In addition, both gene expressions were high in OSCC and OLP and low in controls, which is consistent with the results of our study in which the gene expression of Cav-1 gradually increased in OSCC.

Among the studies reviewed in the present work, only Chen and Yang performed genetic testing on the samples. Given that the study of gene expression using the qRT-PCR method is more accurate than directly measuring the amount of marker in the tissue or observing its staining pattern and that it depends less on the competency of the examiner, we expect the results of the present work to be more 
reliable. In contrast to IHC, however, this method cannot detect the position of the gene and the expression of markers in the cell due to the loss of cell structure in the laboratory. Therefore, the best and most accurate method is to use PCR and IHC. A gradual increase in gene expression with increasing stage and degree of OSCC pathology was only observed in Cav-1, while the Cat-B expression decreased in grade 1 compared to grade 2 , which could not be justified.

Overall, although in our study the gene expressions of Cat-B and Cav-1 increased in OLP and OSCC, we observed a different and contradicting result for Cav-1. This can be explained by noting that in some genes like Cav-1, the multiple function is related to different parts of the structure of this marker that can result in either susceptibility or resistance to cancer (7). The Tye-13 domain of Cav-1 causes cells to grow, while its scaffolding domain suppresses the tumor (9).

\section{Conclusion}

Increased gene expressions of Cat-B and Cav-1 in OSCC were associated with increase in the stage and grade of malignancy. Therefore, by examining these expressions, we can predict the biological behavior of OSCC as well as the possibility of malignant transformations of OLP. Since the gene expressions of Cat-B and Cav-1 increase in OLP and OSCC compared to controls, it can be concluded that these two markers are involved in both the carcinogenesis of OSCC and precancerous pathogenesis such as OLP.

\section{Declarations}

\section{Acknowledgment}

This study was carried out in the Department of Dental and Maxillofacial Diseases as well as in the Pathobiology and Immunology Laboratories of Mashhad dentistry faculty in Iran and was supported by grants from Mashhad university of medical sciences and approved by ethical committee of Mashhad university of medical sciences.

\section{Conflict of Interest}

None.

\section{Author Contributions}

All listed authors have made enough contributions to this study. Negin Samiee did the incisional biopsy of the patients, drafted the manuscript and extracted the data. Atessa Pakfetrat and Zahra Delavarian proposed the initial idea and supervised the project, reviewed and authorized the manuscript. Nooshin Mohtasham and Farnaz Mohajertehran took part in the pathologic evaluation of the samples.

\section{References}

1. Glick M. Burket's oral medicine: PMPH USA; 2015. 
2. Tan G-J, Peng Z-K, Lu J-P, Tang F-Q. Cathepsins mediate tumor metastasis. World journal of biological chemistry. 2013;4(4):91.

3. Pakfetrat A, Basir Shabestari S, Falaki F. Five year clinical and epidemiologic findings of oral lichen planus patients referred to oral medicine department of Mashhad dental school-Iran. Journal of mashhad dental school. 2008;32(3):195-8.

4. Little JW, Falace D, Miller C, Rhodus NL. Dental Management of the Medically Compromised PatientE-Book: Elsevier Health Sciences; 2017.

5. Yang X, Wei K-j, Zhang L, Pan H-y, Li J, Zhong L-p, et al. Increased expression of Cathepsin B in oral squamous cell carcinoma. International journal of oral and maxillofacial surgery. 2010;39(2):174-81.

6. Routray S. Caveolin-1 in oral squamous cell carcinoma microenvironment: an overview. Tumor Biology. 2014;35(10):9487-95.

7. Jaafari-Ashkavandi Z, Aslani E. Caveolin-1 expression in oral lichen planus, dysplastic lesions and squamous cell carcinoma. Pathology-Research and Practice. 2017;213(7):809-14.

8. Hung KF, Lin SC, Liu CJ, Chang CS, Chang KW, Kao SY. The biphasic differential expression of the cellular membrane protein, caveolin-1, in oral carcinogenesis. Journal of oral pathology \& medicine. 2003;32(8):461-7.

9. Fu P, Chen F, Pan Q, Zhao X, Zhao C, Cho WC-S, et al. The different functions and clinical significances of caveolin-1 in human adenocarcinoma and squamous cell carcinoma. OncoTargets and therapy. 2017;10:819.

10. Van der Meij E, Van der Waal I. Lack of clinicopathologic correlation in the diagnosis of oral lichen planus based on the presently available diagnostic criteria and suggestions for modifications. Journal of oral pathology \& medicine. 2003;32(9):507-12.

11. Thongprasom K, Luangjarmekorn L, Sererat T, Taweesap W. Relative efficacy of fluocinolone acetonide compared with triamcinolone acetonide in treatment of oral lichen planus. Journal of oral pathology \& medicine. 1992;21(10):456-8.

12. Chen M-K, Su S-C, Lin C-W, Tsai C-M, Yang S-F, Weng C-J. Cathepsin B SNPs elevate the pathological development of oral cancer and raise the susceptibility to carcinogen-mediated oral cancer. Human genetics. 2012;131(12):1861-8.

13. Satelur KP, Bopaiah S, Bavle RM, Ramachandra P. Role of Cathepsin B as a Marker of Malignant Transformation in Oral Lichen Planus: An Immunohistochemical Study. Journal of clinical and diagnostic research: JCDR. 2017;11(8):ZC29.

14. Kato K, Miyazawa H, Kobayashi H, Noguchi N, Lambert D, Kawashiri S. Caveolin-1 Expression at Metastatic Lymph Nodes Predicts Unfavorable Outcome in Patients with Oral Squamous Cell Carcinoma. Pathology \& Oncology Research. 2020:1-9.

15. Auzair LBM, Vincent-Chong VK, Ghani WMN, Kallarakkal TG, Ramanathan A, Lee CE, et al. Caveolin 1 (Cav-1) and actin-related protein 2/3 complex, subunit 1B (ARPC1B) expressions as prognostic indicators for oral squamous cell carcinoma (OSCC). European Archives of Oto-Rhino-Laryngology. 2016;273(7):1885-93. 
Figures

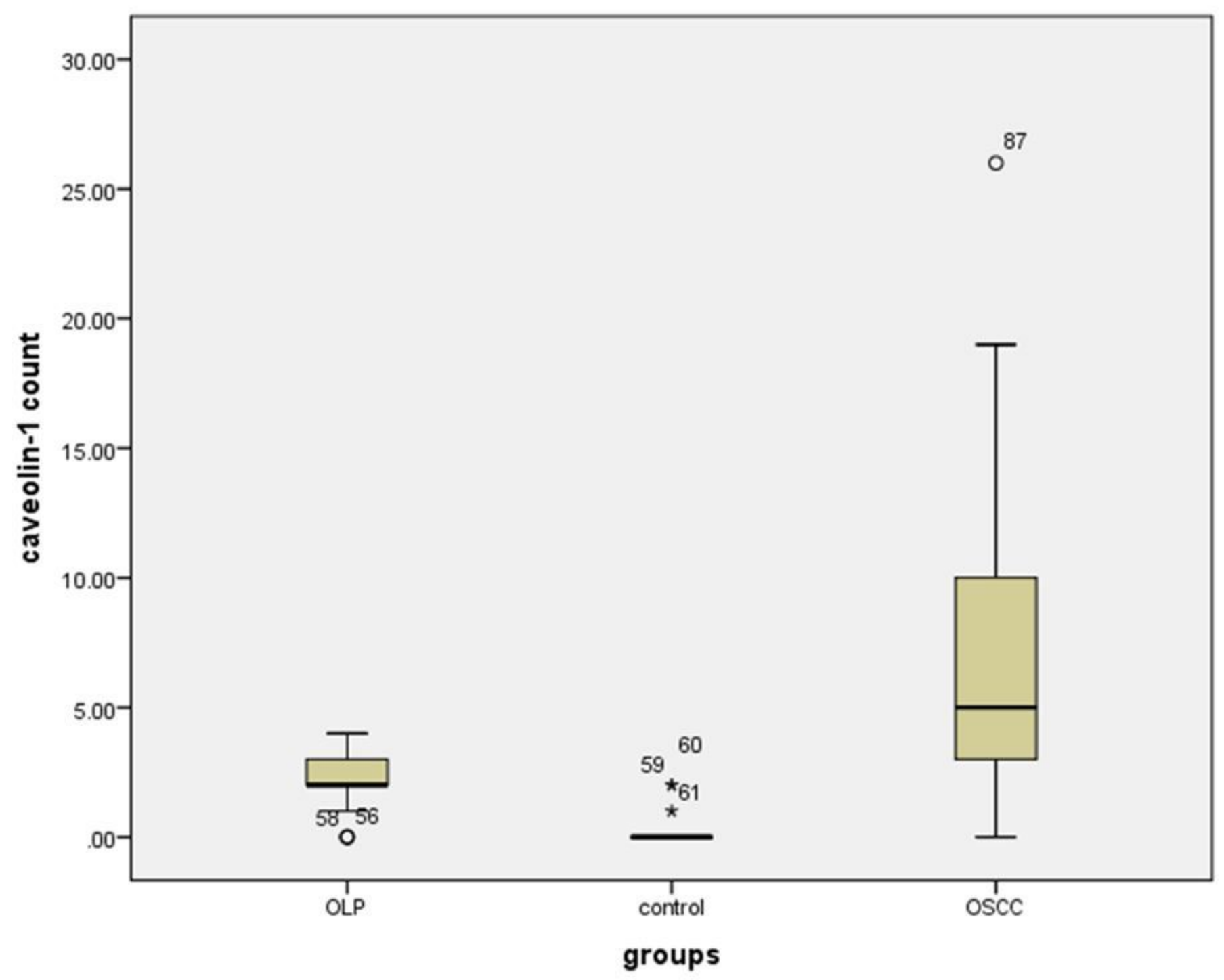

Figure 1

The gene expression of Cav-1 in OLP, OSCC, and controls. 


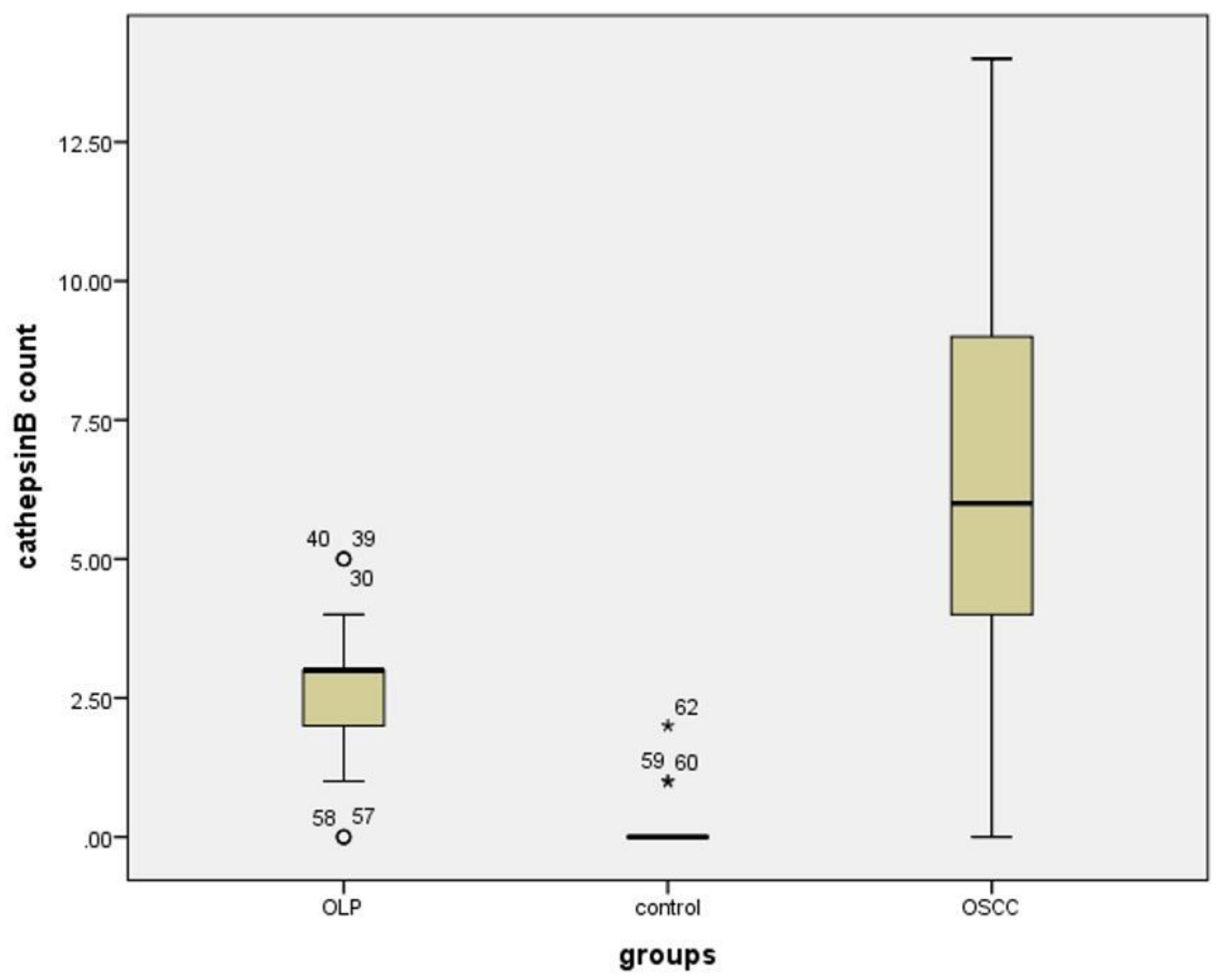

Figure 2

The gene expression of Cat-B in OLP, OSCC, and controls. 